\title{
Review of Color Image Compression using Discrete Wavelet Transform and Block based Image Coding
}

\author{
Sandeep Rai \\ Department of Computer Science \\ and Engineering \\ Technocrats Institute of Technology \\ (Excellence), Bhopal-22, India
}

\author{
Aditi Shrivastava \\ Department of Computer Science \\ and Engineering \\ Technocrats Institute of Technology \\ (Excellence), Bhopal - 22, India
}

\author{
Rajesh Nigam \\ Department of Computer Science \\ and Engineering \\ Technocrats Institute of Technology \\ (Excellence), Bhopal-22, India
}

\begin{abstract}
In this modern era of multimedia, the need of image/video storage and transmission for video conferencing, image and video retrieval, video playback, etc. are increasing at very high rate. As a result, the need for more satisfactory compression technology is always in demand. Modern applications, notwithstanding high pressure proportion, additionally interest for proficient encoding and translating forms, so that to fulfill computational requirement of some continuous applications. Two generally utilized spatial space pressure methods are discrete wavelet change and staggered block truncation coding (BTC).DWT method is used to stationary and non-stationary images and applied to all average pixel value of image. Muli-level BTC is a type of lossy picture pressure system for grayscale pictures. In this, it separates the first pictures into squares and after that a quantizer is utilized to lessen the quantity of dark dimensions in each square yet keeping up a similar mean and standard deviation. In this paper is studied of Multi-level BTCand DWT technique for for gray and color image.
\end{abstract}

\section{Keywords}

DWT, Multi-level, Block Truncation Code (BTC), PSNR MSE, Compression Ratio, Quantizer

\section{INTRODUCTION}

The rising sight and sound innovation and development of GUI based programming have made computerized picture information a characteristic piece of present day life. At the point when a 2-D light power work is inspected and quantized to make an advanced picture, the measure of information produced might be expansive in volume that it results intremendousstorage, processing and communicationrequirements. Therefore, the hypothesis of information pressure turns out to be increasingly more significant for lessening the information repetition to spare more equipment space and transmissionbandwidth [1].

In computer science and information theory,data compression is the process of encoding informationusing less number of bits or some other information bearingunits. Pressure is valuable as it decreases the utilization of costly assets, for example, hard plate spaceor transmission data transfer capacity [3]. BTC is a basic and quick lossy pressure method for dim scale pictures. The fundamental thought of BTC $[2,4]$ is to perform minute saving quantization for squares of pixels. The info picture is isolated into non-covering squares of pixels of sizes $4 \times 4,8 \times 8$, etc. Mean and standard deviation ofthe squares are determined. Mean is considered as thethreshold and recreation esteems are resolved usingmean and standard deviation [5].
At that point a bitmap of the square isderived dependent on the estimation of the limit which is thecompressed or encoded picture. Utilizing the reconstructionvalues and the bitmap the reproduced picture is generatedby the decoder. Along these lines in the encoding procedure, BTCproduces a bitmap, mean and standard deviation for eachblock. It gives a pressure proportion of 4 and bit rate of 2 bitsper pixel when a $4 \times 4$ square is considered $[3,5]$. This methodprovides a decent pressure absent much debasement onthe recreated picture. Be that as it may, it demonstrates a few antiques likestaircase impacts or shoddiness close to the edges. Because of itssimplicity and simple execution, BTC has picked up wideinterest in its further improvement and application for imagecompression [6].

To improve the nature of the reconstructedimage and for the better pressure effectiveness severalvariants of BTC have been created amid the last manyyears.Absolute Moment Block Truncation Coding (AMBTC) [7, 10] jam the higher mean and lower mean of each square and utilize this amount to quantize yield. AMBTC gives preferred picture quality over picture pressure utilizing BTC. Also, the AMBTC is very quicker contrasted with BTC. The calculation is computationally quicker in light of the fact that it includes straightforward expository formulae to figure the parameters of the edge highlight in a picture square $[8,9]$. Remade pictures are of good quality as per human perceptual experience. The calculation speaks to the picture as far as its parallel edge map, mean data, and the force data on the two sides of the edges [10].

\section{LITERATURE SURVEY}

Transform domain downward conversion (TDDC)for image coding is usually implemented by discarding somehighfrequency components from each transformed block. As a result of whicha square of less coefficients is shaped and furthermore lower pressure cost is accomplished and this occurs so on the grounds that in this the coding is done of just a hardly any low-recurrence coefficients. In this paper, center around the plan of another TDDC-based coding technique by utilizing as proposed introduction pressure coordinated separating (ICDF) and mistake repaid scalar quantization (ECSQ), prompting the pressure subordinate TDDC (CDTDDC) based coding. All the more explicitly, ICDF is first used to change over each $16 \times 16$ macroblock into a $8 \times 8$ coefficient square. At that point, this coefficient square is packed with ECSQ, bringing about a littler pressure mutilation for those pixels that situate at some particular places of a large scale square [1]. The select these situations as indicated by the 4:1 uniform sub-examining cross section and utilize the pixels situating at them to reproduce the entire full scale obstruct through an interpolation.Experimental results show that applying proposed CDTDDC-based coding 
to pack still pictures can accomplish a huge quality increase over the current pressure strategies.

Shading and multispectral picture compression using Enhance block truncation code is proposed. These techniques are based on standard deviation and mean [2]. This technique is applied to satellite image and reshapes the satellite image. The satellite image is divided into various sub-blocks. After calculate mean values, all number of pixel in sub-block are compared to the mean and according to the mean all pixel value is replaced by binary number. Finally MSE, PSNR and compression ratio are calculated for the Enhance block truncation code for satellite image.

With the proceeding with development of present day correspondence innovations, interest for picture information pressure is expanding quickly. Methods for accomplishing information pressure can be partitioned into two fundamental methodologies: spatial coding and Transform coding [3, 4]. This exploration paper shows a proposed technique for the pressure of computerized pictures utilizing half breed pressure strategy dependent on Block Truncation Coding (BTC) and Walsh Hadamard Transform (WHT). The target of this cross breed approach is to accomplish higher pressure proportion by applying BTC and WHT. A few grayscale test pictures are utilized to assess the coding efficencyand execution of the cross breed technique and contrasted and the BTC and WHT individually [5]. It is for the most part demonstrated that the proposed technique gives better results.Processing reliance in the ordinary calculation is evacuated by dividing the information picture and changing neighboring reference pixel arrangement. Trial results demonstrate that the parallel execution definitely lessen handling time by $6 \sim 7$ occasions with noteworthy visual quality improvement.

In this paper, picture pressure assumes indispensable job in sparing memory extra room and sparing time while transmissionimages over system. The shading and multispectral picture is considered as information picture for the picture pressure [6, 7]. The proposed system with Enhanced Block Truncation Coding [EBTC] is connected on part of shading and multispectral picture. The part picture is partitioned into different sub squares. Subsequent to assessing mean qualities, the quantity of bits can be decreased by Enhanced Block Truncation Coding. At last, pressure proportion table is created utilizing the parameters, for example, MSE, SNR and PSNR [8]. The proposed strategy is executed through standard shading and multispectral pictures utilizing MATLAB Version 8.1 R2013a.

Block truncation committal to composing (BTC) has been thought of very affordable pressure system for a long time. In addition, this strategy can give amazing preparing proficiency by misusing the nature parallelism preferred standpoint of the spot dispersion, and fantastic picture quality can likewise be offered through co-enhancing the class framework and diffused lattice of the dab dissemination $[9,10]$. As per the trial results, the proposed DDBTC is better than the previous mistake diffused BTC regarding different target picture quality evaluation techniques just as handling proficiency. With the developing media innovation, picture information has been produced at high volume. It is along these lines critical to decrease the picture record sizes for capacity and powerful correspondence [11]. Square Truncation Coding (BTC) is a lossy picture pressure system which utilizes minute saving quantization strategy for packing computerized dark dimension pictures. Despite the fact that this technique holds the visual nature of the recreated picture with great pressure proportion, it demonstrates a few ancient rarities like staircase impact, shoddiness, and so on close to the edges $[12,13]$. A lot of cutting edge BTC variations announced in writing were considered and it was discovered that however the pressure proficiency is great, the nature of the picture must be improved. A changed Block Truncation Coding utilizing maxmin quantizer (MBTC) is proposed in this paper to beat the previously mentioned disadvantages. In the ordinary BTC, quantization is done dependent on the mean and standard deviation of the pixel esteems in each square. In the proposed technique, rather than utilizing the mean and standard deviation, a normal estimation of the most extreme, least and mean of the squares of pixels is taken as the edge for quantization.

Table 1: Summary of Literature Review

\begin{tabular}{|c|c|c|c|}
\hline Title & $\begin{array}{c}\text { Publicat } \\
\text { ion and } \\
\text { Year }\end{array}$ & Methodology & Parameter \\
\hline $\begin{array}{c}\text { Compression- } \\
\text { dependent } \\
\text { Transform } \\
\text { Domain } \\
\text { Downward } \\
\text { Conversion for } \\
\text { Block-based } \\
\text { Image Coding } \\
\text { [1] }\end{array}$ & $\begin{array}{c}\text { IEEE } \\
\text { Trancati } \\
\text { on, } 2018\end{array}$ & $\begin{array}{c}\text { Image } \\
\text { compression } \\
\text { using CDTDDC } \\
\text { based codingto } \\
\text { compress both } \\
\text { gray and color } \\
\text { image. }\end{array}$ & $\begin{array}{c}\text { PSNR }=38.7 \\
\mathrm{~dB} \text { for Lena, } \\
\mathrm{PSNR}=37.4 \\
\mathrm{~dB} \text { for flower, } \\
\mathrm{PSNR}=37.3 \\
\mathrm{~dB} \text { for } \\
\text { butterfly } \\
\text { image }\end{array}$ \\
\hline $\begin{array}{c}\text { A Cost and } \\
\text { Power Efficient } \\
\text { Image } \\
\text { Compressor } \\
\text { VLSI Design } \\
\text { with Fuzzy } \\
\text { Decision and } \\
\text { Block Partition } \\
\text { for Wireless } \\
\text { Sensor } \\
\text { Networks [2] }\end{array}$ & $\begin{array}{c}\text { IEEE } \\
\text { Trancati } \\
\text { on, } 2017\end{array}$ & $\begin{array}{c}\text { Image } \\
\text { compression } \\
\text { using fuzzy } \\
\text { decision based } \\
\text { plockprtition } \\
\text { technique }\end{array}$ & $\begin{array}{c}\text { PSNR = } \\
35.81 \text { for } \\
\text { Lena, PSNR } \\
=36.02 \mathrm{~dB} \\
\text { for Airplane, } \\
\text { PSNR = } \\
\text { 34.78 dB for } \\
\text { Peppers } \\
\text { image }\end{array}$ \\
\hline $\begin{array}{l}\text { RGBW Image } \\
\text { Compression } \\
\text { by Low- } \\
\text { Complexity } \\
\text { Adaptive } \\
\text { Multi-Level } \\
\text { Block } \\
\text { Truncation } \\
\text { Coding [3] }\end{array}$ & $\begin{array}{c}\text { IEEE } \\
\text { Trancati } \\
\text { on, } 2016\end{array}$ & $\begin{array}{c}\text { Image } \\
\text { compression } \\
\text { using adaptive } \\
\text { multi-level BTC } \\
\text { technique }\end{array}$ & $\begin{array}{c}\text { PSNR = } \\
34.04 \text { for } \\
\text { Lena, PSNR } \\
=33.89 \mathrm{~dB} \\
\text { for Pepper, } \\
\text { PSNR = } \\
\text { 32.78 dB for } \\
\text { Baboon } \\
\text { image }\end{array}$ \\
\hline $\begin{array}{c}\text { Color and } \\
\text { Multispectral } \\
\text { Image } \\
\text { Compression } \\
\text { using Enhanced } \\
\text { Block } \\
\text { Truncation } \\
\text { Coding [E- } \\
\text { BTC] Scheme } \\
\text { [4] }\end{array}$ & $\begin{array}{c}\text { IEEE } \\
\text { Conferen } \\
\text { ce, } 2016\end{array}$ & $\begin{array}{c}\text { Image } \\
\text { compression } \\
\text { using enhanced } \\
\text { Block } \\
\text { Truncation Code }\end{array}$ & $\begin{array}{c}\text { PSNR = } \\
32.81 \text { for } \\
\text { Lena, PSNR } \\
=31.72 \mathrm{~dB} \\
\text { for Airplane, } \\
\text { PSNR = } \\
30.78 \mathrm{~dB} \text { for } \\
\text { Peppers } \\
\text { image }\end{array}$ \\
\hline Improved & IEEE & Image & PSNR $=$ \\
\hline
\end{tabular}




\begin{tabular}{|c|c|c|c|}
\hline $\begin{array}{c}\text { Block } \\
\text { Truncation } \\
\text { Coding Using } \\
\text { Optimized Dot } \\
\text { Diffusion [5] }\end{array}$ & $\begin{array}{l}\text { Trancati } \\
\text { on, } 2014\end{array}$ & $\begin{array}{c}\text { compression } \\
\text { using dot } \\
\text { diffusion method }\end{array}$ & $\begin{array}{c}28.90 \text { for } \\
\text { Lena, PSNR } \\
=31.22 \mathrm{~dB} \\
\text { for Pirate } \\
\text { mage }\end{array}$ \\
\hline $\begin{array}{l}\text { Modified BTC } \\
\text { Algorithm for } \\
\text { Gray Scale } \\
\text { Images using } \\
\text { max-min } \\
\text { Quantizer [6] }\end{array}$ & $\begin{array}{c}\text { IEEE } \\
\text { Conferen } \\
\text { ce, } 2013\end{array}$ & $\begin{array}{c}\text { Gray image } \\
\text { compression } \\
\text { using BTC } \\
\text { technique }\end{array}$ & $\begin{array}{c}\text { PSNR }= \\
28.03 .81 \text { for } \\
\text { Lena, PSNR } \\
=29.42 \mathrm{~dB} \\
\text { for Pepper } \\
\text { Image }\end{array}$ \\
\hline
\end{tabular}

\section{PROBLEM IDENTIFICATION}

Uncompressed multimedia (audio, video and graphics) data requires considerable storage capacity and transmission bandwidth. Compression, is an efficient technique for massive storage capacity, reduces transmission bandwidth, and transmission time needed to transmit data $[12,14]$. Regardless of quick advancement in mass-stockpiling limit, processor speeds, and computerized correspondence framework execution, interest for information stockpiling limit and information transmission data transfer capacity keeps on overwhelming the abilities of accessible innovations. The ongoing development of information serious mixed media based web applications not just continued the requirement for increasingly productive approaches to encode signals vital to capacity and correspondence innovation. Picture pressure is the system to lessen the redundancies in information portrayal so as to diminish information stockpiling necessities and subsequently correspondence costs. Lessening the capacity prerequisite is comparable to expanding the limit of the capacity medium and consequently correspondence data transfer capacity $[11,15,16]$. Along these lines the improvement of proficient pressure methods will keep on being a plan challenge for future correspondence frameworks and propelled sight and sound applications. Fundamentally, picture is spoken to as a mix of data and repetition [17].

Picture pressure is the system to diminish the redundancies in information portrayal so as to diminish information stockpiling prerequisites and henceforth correspondence costs. Decreasing the capacity prerequisite is identical to expanding the limit of the capacity medium and henceforth correspondence transfer speed [18, 19]. In this manner the improvement of productive pressure strategies will keep on being a structure challenge for future correspondence frameworks and propelled mixed media applications. From the writing audit infer that the recently connected system is ICDF. Past strategy was partitioned the picture into four section and ICDF method was working just initial segment different parts as zero. The prooblem emerge was the loss of nature of picture in term of pinnacle peak signal to noise ratio (PSNR) and increment the mean square error (MSE).

\section{PROPOSED METHODOLOGY}

Transmission and storage of raw images require huge quantity of disk space. Hence, there is an urgent need to reduce the size of image before sending or storing. The most ideal answer for the issue is to utilize pressure techniques where the pressure of information on computerized pictures are made to diminish superfluity and excess of the picture information to have the capacity to proficiently store or transmit information. The vast majority of the current pressure methods utilized have their negatives and an improved system which is quicker, compelling and memory productive can fulfill the necessities of the client.

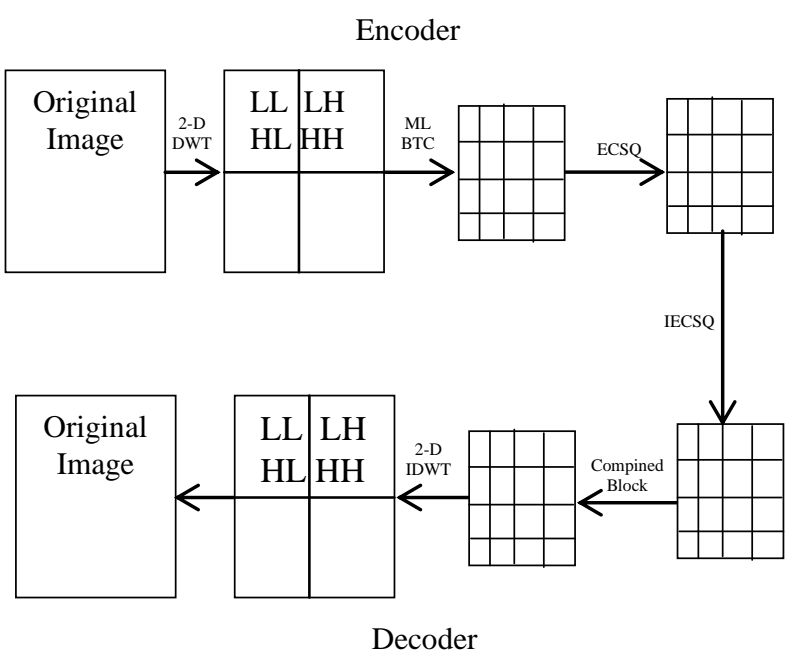

Figure 1: Proposed Methodology

Picture pressure flourishes to store or transmit the information in a capable mode just as to offer a best picture quality at a predefined bit-rate. Picture pressure should be possible in lossy or lossless mode. Lossless pressure is favored for recorded destinations and principally utilized in medicinal imaging, specialized illustrations, cut workmanship, or funnies. This is because of the presentation of pressure ancient rarities, low piece rates and furthermore on the grounds that the assets can't be impressively spared by utilizing picture pressure strategy. Lossy techniques are particularly reasonable for common pictures, for example, photos in applications where irrelevant loss of devotion is bearable to accomplish an impressive decrease in bit rate. Here appeased following picture quality without much discernment by the watcher is accomplished.

\section{- Discrete Wavelet Transform}

Wavelets are signals which are neighborhood in time and scale and for the most part have a sporadic shape. A wavelet is a waveform of adequately restricted length that has a normal estimation of zero. The term 'wavelet' originates from the way that they coordinate to zero; they wave here and there over the pivot. Numerous wavelets additionally show a property perfect for minimized flag portrayal: symmetry. This property guarantees that information isn't over spoken to. A flag can be deteriorated into many moved and scaled portrayals of the first mother wavelet. A wavelet change can be utilized to disintegrate a flag into part wavelets. When this is done the coefficients of the wavelets can be crushed to evacuate a portion of the subtleties. Wavelets have the incredible favorable position of having the capacity to isolate the fine subtleties in a flag. Small wavelets can be utilized to disconnect fine subtleties in a flag, while exceptionally expansive wavelets can recognize coarse subtleties. Furthermore, there are a wide range of wavelets to look over. Different kinds of wavelets are: Morlet, Daubechies, and so forth [6].

This technique at first separates an image into coefficients called sub-gatherings and after that the ensuing coefficients are differentiated and a farthest point. Coefficients underneath the edge are set to zero. Finally, the coefficients over the farthest point regard are encoded with a mishap less weight methodology. The weight features of a given wavelet premise are fundamentally associated with the general scarceness of the wavelet space depiction for the banner. The thought behind weight relies upon the possibility that the standard 
banner section can be definitely approximated using the going with segments: few conjecture coefficients (at a sensibly picked measurement) and a part of the detail coefficients.

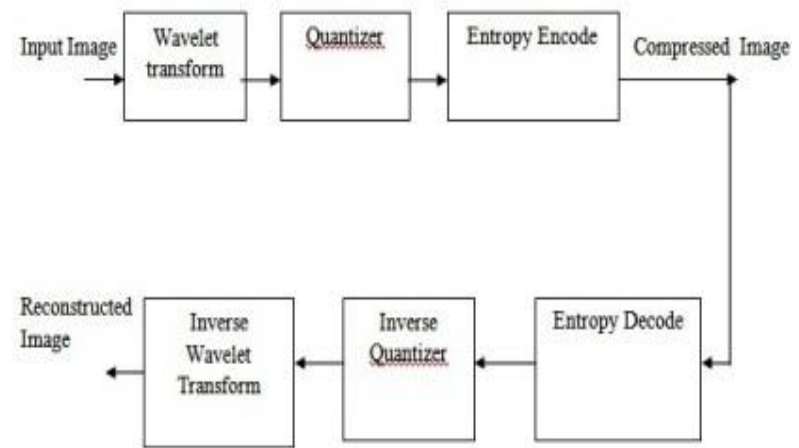

Figure 2: The structure of the wavelet transform based compression.

The steps of compression algorithm based on DWT are described below:

I. Decompose Choose a wavelet; choose a level N. Compute the wavelet. Decompose the signals at level $\mathrm{N}$.

II. Threshold detail coefficients For each level from 1 to $\mathrm{N}$, a threshold is selected and hard thresholding is applied to the detail coefficients.

III. Reconstruct Compute wavelet reconstruction using the original approximation coefficients of level $\mathrm{N}$ and the modified detail coefficients of levels from 1 to $\mathrm{N}$.

\section{- Multi-level Block Truncation Code}

The Encoder and decoder block of the multi-level block truncation code algorithm is shown if figure 2. Encoder part of the proposed algorithm shows that the original image is divided into three parts i.e. R component, $\mathrm{G}$ component and B component. Each R, G, B component of the image is divided into non overlapping block of equal size and threshold value for each block size is being calculated.

Threshold value means the average of the maximum value (max) of ' $\mathrm{k} \times \mathrm{k}$ ' pixels block, minimum value (min) of ' $\mathrm{k} \times \mathrm{k}$ '

pixels block and $m_{1}$ is the mean value of ' $\mathrm{k} \times \mathrm{k}$ ' pixels block. Where $\mathrm{k}$ represents block size of the color image. So threshold value is:

$$
T=\frac{\max +\min +m_{1}}{3}
$$

Each threshold value is passing through the quantization block. Quantization is the process of mapping a set of input fractional values to a whole number. Suppose the fractional value is less than 0.5 , then the quantization is replaced by previous whole number and if the fractional value is greater than 0.5, then the quantization is replaced by next whole number.

$$
\begin{gathered}
H=\frac{1}{p} \sum_{i=1}^{p} W_{i} \quad W_{i}>T \\
L=\frac{1}{q} \sum_{i=1}^{p} W_{i} \quad W_{i} \leq T
\end{gathered}
$$

Wi represent the input color image block, $\mathrm{q}$ is the number of zeros in the bit plane, $\mathrm{p}$ is the number of ones in the bit plane. In the combine block of decoder, the values obtained from the pattern fitting block of individual R, G,B components are combined after that all the individual combined block are merged into a single block . Finally compressed image and all the parameter relative to that image will be obtained.
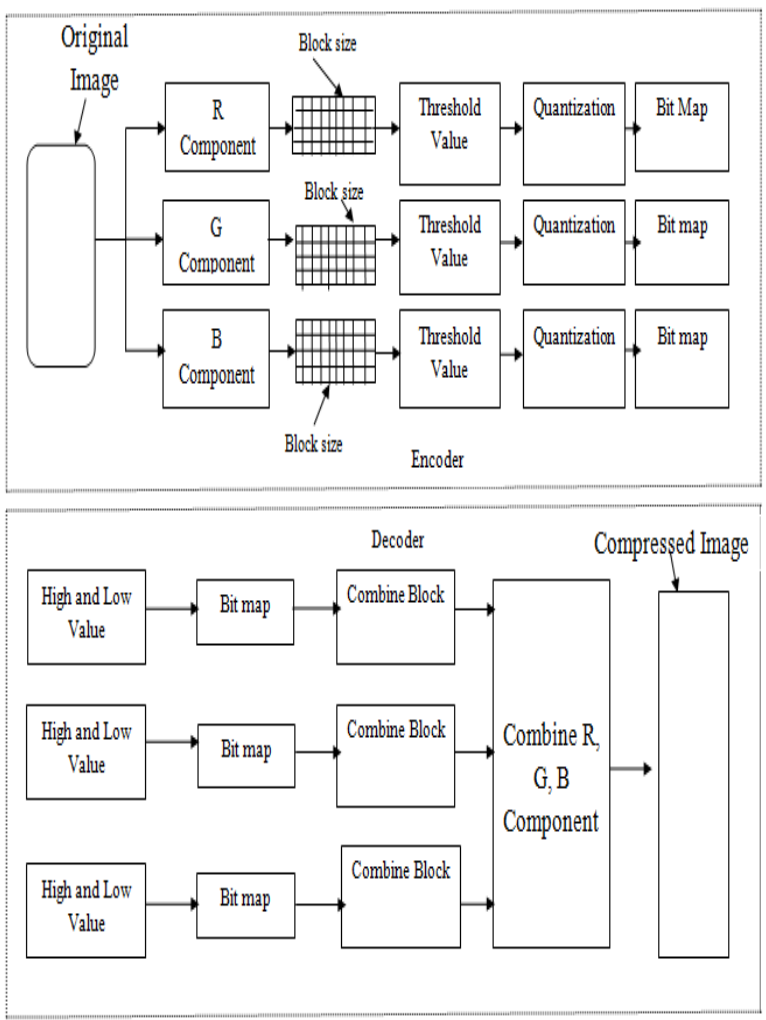

Figure 3: Block Diagram of Proposed Algorithm

Each quantization value is passing through the bit map block. Bit map means each block is represented by ' 0 ' and ' 1 ' bit map. If the Threshold value is less than or equal to the input image value then the pixel value of the image is represent by ' 0 ' and if the threshold value is greater than the input image value then the pixel value of the image is represented by ' 1 '.

Bit map is directly connected to the high and low component of the proposed decoder multi-level BTC algorithm. High $(\mathrm{H})$ and low (L) component is directly connected to the bit map, bitmap converted the ' 1 ' and ' 0 ' pixel value to high and low pixel value and arrange the entire block.

- $\quad$ Error-compensated scalar quantization

The utilization of ICDF in the TDDC-based coding pointsata better addition and a lower pressure cost. In any case, when the pressure occurs, the interjection productivity just as the coding effectiveness will be restricted by the contortion happening on those separated pixels (meant as $\sim \mathrm{x}$ ) that will be utilized for insertion. To take care of this issue, as reason to decrease the entirety of square blunder (SSE) bending of $\sim$ xas much as conceivable by means of controlling the quantization mistake of the changed large scale square dependent on a blunder repaid scalar quantization (ECSQ).

\section{CONCLUSION}

Such method is suitable in situations where image or image is compressed once but decoded frequently. It is clear that the decoding time due to spatial domain based compression is much less than that of the sub-band compression techniques. In his paper the study of discretewavelet transform, mult-level block truncation code and error-compensated scalar quantizationtechnique.Further work of this paper is to 
implement proposed algorithm in MATLAB software and compare result in implementation paper

\section{REFERENCES}

[1] Shuyuan Zhu, Zhiying He, XiandongMeng, Jiantao Zhou and Bing Zeng, "Compression-dependent Transform Domain Downward Conversion for Block-based Image Coding”, IEEE Transactions on Image Processing, Volume: 27, Issue: 6, June 2018.

[2] Shih-Lun Chen and Guei-Shian Wu, "A Cost and Power Efficient Image Compressor VLSI Design with Fuzzy Decision and Block Partition for Wireless Sensor Networks", IEEE Sensors Journal, Volume: 17, Issue: 15, Aug.1, 12017.

[3] Sunwoong Kim and Hyuk-Jae Lee, "RGBW Image Compression by Low-Complexity Adaptive Multi-Level Block Truncation Coding", IEEE Transactions on Consumer Electronics, Vol. 62, No. 4, November 2016.

[4] C. Senthilkumar, "Color and Multispectral Image Compression using Enhanced Block Truncation Coding [E-BTC] Scheme", accepted to be presented at the IEEE WiSPNET, PP. 01-06, 2016 IEEE.

[5] Jing-Ming Guo, Senior Member, IEEE, and Yun-Fu Liu, Member, IEEE, "Improved Block Truncation Coding Using Optimized Dot Diffusion", IEEE Transactions on Image Processing, Vol. 23, No. 3, March 2014.

[6] Seddeq E. Ghrare and Ahmed R. Khobaiz, "Digital Image Compression using Block TruncationCoding and Walsh Hadamard Transform Hybrid Technique", 2014 IEEE 2014 International Conference on Computer, Communication, and Control Technology (I4CT 2014), September 2 - 4, 2014 - Langkawi, Kedah, Malaysia.

[7] Jayamol Mathews, Madhu S. Nair, "Modified BTC Algorithm for Gray Scale Images using max-min Quantizer”, 978-1-4673-5090-7/13/\$31.00 @2013 IEEE.

[8] Harihara Santosh, U. V. S. Sitarama Varma, K. S. K Chaitanya Varma, Meena Jami, V. V. N. S Dileep, "Absolute Moment Block Truncation Coding For Color Image Compression," International Journal of Innovative Technology and Exploring Engineering (IJITEE), Volume-2, Issue-6, PP. 53-59, May 2013.

[9] Yun-Ho Ko, Jin-Hyung Kim, Si-Woong Lee, and HyunSoo Kang, "Dual Block truncation Coding for
Overdriving of Full HD LCD Driver," IEEE Tarnation of Image Processing, vol. 12, No. 08, PP. 01-07, 2012 IEEE.

[10] Jing-Ming Guo and Yun-Fu Liu, "High Capacity Data Hiding for Error-Diffused Block Truncation Coding," IEEE Transactions on Image Processing, Vol. 21, No.12, PP.4808-4817, December 2012.

[11] Doaa Mohammed, Fatma Abou-Chadi, "Image Compression Using Block Truncation Coding," Multidisciplinary Journals in Science and Technology, Journal of Selected Areas in Telecommunications, PP.913, February Edition, 2011.

[12] Anil and K. V. Karthik, "A Modified Three Level Block Truncation Coding _or Image Compression", International Conference on Pattern Analysis and Intelligent Robotics, PP.31-35, June 2011 IEEE.

[13] Shashikumar.S, Arpana Parakale, Bharamgonda Madhuri Mahavir, Bharamu Ullagaddi, " Image Compression using Absolute Moment Block Truncation Coding", International Conference on Pattern Analysis and Intelligent Robotics, PP.97-102, June 2011, Putrajaya, Malaysia.

[14] Yung-Chen Chou and Hon-Hang Chang, "A Data Hiding Scheme for Color Image Using BTC Compression Technique," Proc. 9th IEEE International Conference on Cognitive Informatics, PP.845-850, 2010 IEEE.

[15] M. Brunig and W. Niehsen. Fast full search block matching. IEEE Transactions on Circuits and Systems for Video Technology, 11:241 - 247, 2001.

[16] K. W. Chan and K. L. Chan. Optimisation of multi-level block truncation coding. Signal Processing: Image Communication, 16:445 - 459, 2001

[17] Ki-Won Oh and Kang-Sun Choi, "Paralle Implementation of Hybrid Vector Quantizerbased Block Truncation Coding for Mobile Display Stream Compression”, IEEE ISCE 20141569954165

[18] C. C. Chang, H. C. Hsia, and T. S. Chen. A progressive image transmission scheme based on block truncation coding. In LNCS Vol 2105, pages 383-397, 2001.

[19] C. C. Chang and T. S. Chen. New tree-structured vector quantization with closed-coupled multipath searching method. Optical Engineering, 36:1713 - 1720, 1997. 